\title{
BMJ open A qualitative study of GP, NP and patient views about the use of rapid streptococcal antigen detection tests (RADTs) in primary care: 'swamped with sore throats?'
}

\author{
Gerry M Leydon, ${ }^{1}$ Lisa McDermott, ${ }^{1}$ Mike Moore, ${ }^{1}$ Ian Williamson, ${ }^{1}$ \\ F D Richard Hobbs, ${ }^{2}$ Tessa Lambton, ${ }^{1}$ Rebecca Cooper, ${ }^{1}$ Hugo Henderson, ${ }^{1}$ \\ Paul Little, on behalf of the PRISM Investigators
}

To cite: Leydon GM, McDermott L, Moore M, et al. A qualitative study of GP, NP and patient views about the use of rapid streptococcal antigen detection tests (RADTs) in primary care: 'swamped with sore throats?'. BMJ Open 2013;3 e002460. doi:10.1136/ bmjopen-2012-002460

- Prepublication history for this paper are available online. To view these files please visit the journal online (http://dx.doi.org/10.1136/ bmjopen-2012-002460).

Received 6 December 2012 Accepted 5 February 2013

This final article is available for use under the terms of the Creative Commons Attribution Non-Commercial 2.0 Licence; see http://bmjopen.bmj.com

${ }^{1}$ Department of Primary Care and Population Sciences, University of Southampton, Southampton, UK ${ }^{2}$ Department of Primary Care, Oxford University, Oxford, UK

Correspondence to Dr Geraldine Leydon; G.M.Leydon@soton.ac.uk

\section{ABSTRACT}

Objective: To explore patient and healthcare professionals' (HCP) views of clinical scores and rapid streptococcal antigen detection tests (RADTs) for acute sore throat.

Design: Qualitative semistructured interview study.

Setting: UK primary care.

Participants: General practitioners (GPs), nurse practitioners (NPs) and patients from general practices across Hampshire, Oxfordshire and the West Midlands who were participating in the Primary Care Streptococcal Management (PRISM) study.

Method: Semistructured, face-to-face and phone interviews were conducted with GPs, NPs and patients from general practices across Hampshire, Oxfordshire and the West Midlands.

Results: 51 participants took part in the study. Of these, 42 were HCPs (29 GPs and $13 \mathrm{NPs}$ ) and 9 were patients. HCPs could see a positive role for RADTs in terms of reassurance, as an educational tool for patients, and for aiding inexperienced practitioners, but also had major concerns about RADT use in clinical practice. Particular concerns included the validity of the tests (the role of other bacteria, and carrier states), the tension and possible disconnect with clinical assessment and intuition, the issues of time and resource use and the potential for medicalisation of self-limiting illness. In contrast, however, experience of using RADTs over time seemed to make some participants more positive about using the tests. Moreover, patients were much more positive about the place of RADTs in providing reassurance and in limiting their antibiotic use.

Conclusions: It is unlikely that RADTs will have a (comfortable) place in clinical practice in the near future until health professionals' concerns are met, and they have direct experience of using them. The routine use of clinical scoring systems for acute upper respiratory illness also face important barriers related to clinicians' perceptions of their utility in the face of clinician experience and intuition.

\section{ARTICLE SUMMARY}

Article focus

- To explore patients' and healthcare professionals (HCP) views on and experiences of clinical scores and rapid streptococcal antigen detection tests (RADTs) for acute sore throat.

Key messages

- It is unlikely that RADTs will have a place in clinical practice until health professionals' concerns are addressed.

- Clinicians' perceptions of their utility in the face of clinician experience and intuition may serve as a barrier to their use.

- Direct experience is one key way in which practitioner concerns are likely to be allayed.

Strengths and limitations of this study

- The study included general practitioners, nurse practitioners and patients to capture a broad view of the RADT in practice.

- The patient sample size was relatively small and further work could test out findings and generate further insights.

- Three researchers conducted the interviews, but transparent procedures ensured this was not a problem.

\section{BACKGROUND}

Rapid streptococcal antigen detection tests (RADTs) are perhaps the most commonly used near patient test for acute respiratory infections. The RADT is a near patient test which can be used during a consultation to provide an immediate result for the presence of GABHS (group A $\beta$-haemolytic Streptococcus, a bacterial microorganism involved in acute sore throat), to provide a basis for the prescription of antibiotics. According to the NICE Guidelines (National Institute for Clinical Excellence, 
2008) patients with acute sore throat/pharyngitis/tonsilitis should be considered for an immediate antibioticprescribing strategy if they meet three or more of the centor criteria (which can indicate the presence of GABHS). They are widely advocated and used internationally in the management of acute pharyngitis, although use of RADTS in the UK is currently limited. ${ }^{1-3}$ However, there are concerns raised about the use of RADTs based on anecdotal evidence that suggests the procedure may be time consuming, increase patient expectation for the test, and result in increased levels of patients consulting with a sore throat. ${ }^{4}$ There is very limited qualitative work regarding patients' and practitioners' use of point of care tests for respiratory infections, and in particular for RADTs. Qualitative work with both patients and health practitioners across Europe for another point of care tests used for lower respiratory tract infection, C reactive protein, suggests issues for practitioners include questionable test performance, problems interpreting results, a detraction from clinical reasoning, costs, time and patients not wanting or demanding, the tests. ${ }^{5}$

The use of clinical scoring methods for acute sore throat is also widely advocated in clinical practice guidance, ${ }^{16-9}$ but we are unaware of any qualitative research to document and explore practitioner or patient perceptions about the use of clinical scores. This study provided a nested qualitative component to the PRISM trial-which compared the use of RADTs and clinical scoring methods with delayed antibiotic prescribing for acute sore throat (the PRISM trial aims to evaluate the efficacy of the RADT). The objective of this study was to explore healthcare practitioner (HCP), (nurse practitioner (NP) and general practitioner (GP)) and patient experiences of and attitudes towards RADTs and clinical scores.

\section{METHODS}

\section{Participants and procedure}

Participants were identified and recruited (JB, JK and $\mathrm{KM}$ ) from a mix of inner city and rural practices across the south and southeast of England. Healthcare practitioners were eligible for inclusion if their practice had consented to take part in the PRISM trial. NPs were eligible for inclusion if they had responsibilities that would involve using an RADT in practice (ie, diagnosing and prescribing for respiratory tract infections). Patients were eligible for inclusion if they had experienced an RADT during a consultation (as part of the PRISM trial). Ethical approval for the study was obtained as part of the PRISM trial (Southampton and South West Hampshire REC, MREC number 06/Q1702/111).

\section{Interviews}

Trained interviewers (TL, RC and $\mathrm{HH}$ ) conducted both face-to-face and telephonic interviews (according to participant preference) with each lasting approximately half an hour. All interviews were audio-recorded and transcribed verbatim in preparation for analysis. Qualitative interviews provided the best method for gathering insights into participants' individual views about and experiences of using RADTS. A semi-structured interview guide (appendix 1) included key topic areas while also providing flexibility to explore unanticipated issues. The interview guide was amended before the final 11 interviews were conducted to include a question about participant experiences of using clinical scoring methods (this topic had been discussed in earlier interviews and was included in the guide to ensure a thorough discussion).

\section{Analysis}

Inductive thematic analysis ${ }^{10}$ was conducted on all transcripts to determine healthcare professionals' and patients' experiences and views on RADTS in practice. Following immersion in the transcripts familiarisation was achieved and patterns and prominent themes which consistently occurred in the data were identified and labelled with codes. Each code label referred directly to the operationalisation of the theme content. A label and full descriptive definition was then provided for each theme. The codes and definitions were refined during a continuing process, which involved themes being linked, grouped, moved, relabelled, added and removed to produce a set of themes and subthemes and a coding manual, which adequately fitted and thoroughly explained the data. The coding was iteratively developed across authors (led by LM and GL) and adjustments made where appropriate based on discussion.

\section{FINDINGS}

\section{Participants}

In total 51 participants took part in the study. Of these, 42 were HCPs (29 GPs and 13 NPs) and 9 were patients. In total, GPs/NPs were recruited from 15 practices across Hampshire, Oxfordshire and the West Midlands. Registered practice population size varied from 3272 to 16596 (table 1). Surgeons were recruited from both inner city and rural locations, with the index of multiple deprivation score (IMD) ranging from 2.7 to 36.9 .

\section{Themes}

Analysis identified five main themes, and a total of 18 subthemes relating to experiences and opinions of using RADTs (table 2).

\begin{tabular}{ll} 
Table 1 Practice demographics & \\
\hline Registered practice population size mean & 4049 \\
Registered practice population size range & $3272-16596$ \\
IMD score mean & 11.55 \\
IMD score range & $2.70-36.92$ \\
England average IMD score & 21.7 \\
England lowest IMD score & 2.6 \\
England highest IMD score & 68.9 \\
\hline IMD, index of multiple deprivation score.
\end{tabular}




\begin{tabular}{|c|c|c|}
\hline Theme & Subtheme & \\
\hline Practicalities & $\begin{array}{l}\text { Benefits } \\
\text { Concerns }\end{array}$ & $\begin{array}{l}\text { Reduces antibiotic prescribing } \\
\text { Easy to use } \\
\text { Useful for inexperienced staff } \\
\text { Time concerns } \\
\text { Cost concerns }\end{array}$ \\
\hline Accuracy of diagnosis & $\begin{array}{l}\text { Benefits } \\
\text { Concerns }\end{array}$ & $\begin{array}{l}\text { Confirmation of diagnosis } \\
\text { Conflict of opinion } \\
\text { - Identification of carriers } \\
\text { Alternative bacteria not detected }\end{array}$ \\
\hline Patient outcomes & $\begin{array}{l}\text { Benefits } \\
\text { Concerns }\end{array}$ & $\begin{array}{l}\text { Reassurance for patient } \\
\text { Education tool for patient } \\
\text { Medicalisation concerns }\end{array}$ \\
\hline Experience of using clinical scores & $\begin{array}{l}\text { Benefits } \\
\text { Concerns }\end{array}$ & $\begin{array}{l}\text { Useful for inexperienced practitioners } \\
\text { Unnecessary for experienced practitioners } \\
\text { Time consuming }\end{array}$ \\
\hline RADT trial participation & & $\begin{array}{l}\text { Trial participation increases positive views. } \\
\text { Negative perceived views of non-trial practitioners } \\
\text { Positive views of trial practitioners }\end{array}$ \\
\hline Patient views & & $\begin{array}{l}\text { Reassured by test } \\
\text { No change on GP attendance } \\
\text { Preference for no antibiotics }\end{array}$ \\
\hline
\end{tabular}

In the following sections we describe each theme in turn, using exemplary quotations for illustrative purposes.

\section{Practicalities}

When invited to discuss their practical experiences of the rapid tests, practitioners' views highlighted five key subthemes, all relating to the practicalities of RADTs.

\section{Reduces antibiotic prescribing}

In terms of positive practical feedback, a few practitioners in the trial reported a reduction in their antibiotic prescribing rates for sore throat as a result of using the RADTs.

It (use of antibiotic) has substantially decreased since I started the trial. (Int. 43; GP)

\section{Easy to use}

Despite concerns about the tests increasing consultation lengths (reported in a later section), practitioners reported that the tests were easy to use once they were familiar with them.

It was not that it was a particularly difficult test, it was just the first time you use something you need to know what to do. (Int. 29; GP)

\section{Useful for inexperienced practitioners}

In terms of the practicalities or perhaps skill of appropriate prescribing, some of the more experienced interviewees suggested that the tests could support prescribing decisions for new or inexperienced staff members.
If you're in a teaching or training practice, then it's useful, to get the clinicians to learn. (Int. 48; GP)

\section{Time concerns}

Perhaps unsurprisingly, given the time pressures and short consultation times in general practice and the challenges of incorporating new practices within them, time concerns were common. The RADTs were described as taking up too much time during a consultation. However, practitioners did acknowledge that as the RADT use in this instance formed part of a trial, some of the additional time involved with explaining the study had added considerably to the burden and outside of a trial context use of the test may not be quite as time consuming.

It's the additional time that's needed to do it, is the major problem. (Int. 47, GP)

Usually the longest bit is the patient reading through all the information about the test. (Int. 30; GP)

Practitioners who took part in the trial also reported that their concerns relating to time constraints were reduced once they had started using the test and were clearer on what to do.

...my first thought when the girls (trial researchers) came to talk to us about it was, oh gosh, how long is it going to take? And then once you realize that if you were doing it regularly you could do it much quicker and that's not a problem. (Int. 25; GP) 


\section{Cost concerns}

The majority of practitioners were concerned about the cost implications of using the tests. In particular, participants were worried that RADTS would increase the expenditure of the surgery.

I don't think we would use them, if there was a cost issue. Why do I say that? Because all our other pathology is free. (Int. 26; GP)

\section{Accuracy of diagnosis}

One of the key potential benefits of using rapid tests in practice is the promise of improving the accuracy of diagnosis. Four subthemes related to the accuracy of diagnosis when using the RADT were identified, and each is taken in turn.

\section{Confirmation of diagnosis}

When congruent with a practitioner's diagnosis or management disposal, the rapid test result could work to reassure the practitioner and confirm their opinion of the diagnosis.

It's been reassuring and it reinforces my credibility of making a clinical judgement. (Int. 27; GP)

\section{Conflict of opinion}

In contrast, practitioners reported that their treatment decisions were rendered difficult when there was a conflict between the RADT results and their opinion of the most likely diagnosis.

If it's a negative test and that persons got clinical signs of a bacterial sore throat...you then have to say, err, what do I do here? (Int. 43; GP)

I haven't yet evolved, because it's not available to me...a way of handling a positive test that I actually don't want to treat. (Int. 42; GP)

This resonates with other supported decisions in general practice, such as the use of severity measures for the diagnosis and management of depression (PHQ9/ HADs, Patient Health Questionnaire/Hospital Anxiety and Depression Scale), where GPs have described a careful weighing of the measured evidence versus their GP intuition based on experience. ${ }^{11}$

\section{Identification of carriers}

Practitioners reported concern that the RADT would produce a positive test result for patients who were only carriers for GABHS, and could result in an unnecessary prescription of antibiotics.

...I think they're quite limited really because, because of the fact that streptococcal, you know, that strep is carried so widely anyway... (Int. 15; GP)
Failure to detect alternative bacteria

Practitioners were also concerned that the RADT cannot detect all forms of bacteria.

This test just checks for streptococcal, it doesn't check for the other bacteria. (Int. 46; GP)

I mean it doesn't cover everything does it? It just covers some of the streps doesn't it...don't know how many it covers. (Int. 29; GP)

\section{Patient outcomes}

Practitioners were invited to reflect on the impact the rapid tests might have on patient outcomes. Three subthemes referring to patient-related outcomes were identified. Again each theme is described in turn.

\section{Reassurance for the patient}

Linked to the views that a test score can enhance the credibility of a treatment disposal, practitioners described how the RADT might quickly provide reassurance for patients in the consultation.

I think... they find it very reassuring, as it's so rapid. (Int. 28; GP)

You've got your answer...and you can reassure the patient that you've got the answer, rather than what we tend to say is 'well, this may or may not be. (Int. 51; NP)

\section{Education tool for patient}

In a related fashion, interviewees described how they had used the RADT as a tool to assist in patient education. More particularly, such tests could be used to explain why antibiotics were not always necessary for sore throat.

It (RADT) helps us to explain to patients why we're not prescribing for a condition that, hopefully, is going to be self-limiting. (Int. 47; GP)

You are also able to enter into the discussion with them about the, you know, the supposed benefits of antibiotics, the fact they won't work for everything and it allows you to open this door or viral versus bacterial. (Int. 14; GP)

\section{Medicalisation concerns}

Practitioners also reported a concern that the increased availability of RADTS could lead to patients viewing all sore throats as more serious and thereby increase consultations owing to the demand for the test.

I think people would be, oh, and when I went to the GP surgery they did this special test and I think...if you were a surgery who were offering it you'd be swamped with sore throats, and if you were a surgery who weren't offering it, you'd be swamped with, when my friend up the road gets a special test. So, yeah, I think it might medicalise...something that will go away on its own anyway. (Int. 04; GP) 
The problems come when people then start to think they have to have it done every time. (Int. 27; GP)

\section{Experience of using clinical scores}

The last 11 interviewees were asked to discuss their views and experiences on the use of the clinical score. None of the practitioners reported using the clinical scoring method prior to taking part in the trial. Although there were some positive perceptions of the scoring, the majority of comments and views on the scores were negative. Three subthemes relating experiences of using the clinical scores were identified and these are described in the following section.

\section{Useful for inexperienced practitioners}

Although most practitioners felt that the scoring was unnecessary for experienced members of staff, it was consistently reported that the clinical score did have a 'place within primary care' as a useful educational tool for inexperienced practitioners.

If you're a teaching or training practice, then it's useful to get the clinicians to learn and think about this in a much more systematic manner. (Int. 48; GP)

\section{Unnecessary for experienced practitioners}

In contrast, the most common complaint regarding the clinical scores was that they were unnecessary for an experienced practitioner. GPs described how the questions that form part of the score should be asked during the history taking phase and the additional documentation of these was unnecessary. However, it was noted that NPs did not support this view and felt that the scores would be a useful tool.

One doesn't want to complicate incredibly minor medicine with tools and scanning on bits of paper. (Int. 44; GP)

There's scores for everything at the moment and I think sometimes, you know, if I'm honest, you don't need a doctor to be scoring things. (Int. 47; GP)

\section{Time consuming}

Some practitioners felt that the clinical scoring was too time consuming to fit into usual practice. In particular, it was the time taken to document the score, as opposed to conducting the score itself which was viewed as a problem.

It's very time consuming, you know, you're whizzing through a busy surgery...one would hope one knew the right questions to ask. (Int. 44; GP)

\section{RADT trial participation}

All participants were invited to discuss their views and opinions following participation in the RADT trial. Three subthemes were identified.
Trial participation increases positive views

Practitioners described how their views about RADTs had changed positively since being in the trial.

...And then once you realize that if you were doing it regularly you could do it much quicker and that's not a problem. (Int. 25; GP)

\section{Reluctance of non-trial practitioners}

Practitioners speculated that GPs and NPs who had not been part of the PRISM trial would be reluctant to use the RADT.

My worry is that if you try to sell it to people who have not been part of the PRISM trial, they won't do the test at all. (Int. 26; GP)

\section{Positive views of trial practitioners}

In contrast, practitioners who had taken part in the trial reported that in general they would use the RADT in general practice, if available.

...by doing the rapid test, you are (likely) to have less consultations and repeat consultations and that again gives me a personal benefit. (Int. 28; GP)

\section{Patient views}

Three subthemes relating to patient experiences with the RADT emerged.

\section{Reassured by test}

Patients described feeling reassured with their diagnosis and the treatment option that had been provided owing to having the RADT, which was congruent with the practitioner views on the tests providing reassurance.

$$
\begin{aligned}
& \text {...it was quite reassuring being the patient and having } \\
& \text { the right test for the right medicine... (Int. 32; patient) }
\end{aligned}
$$

\section{No change on GP attendance}

Patients reported that the provision of the RADT would not influence their decision to attend the GPs for a sore throat.

I would still see how it went before I sought medical advice...I wouldn't just run straight to the doctors. I would wait, see how it developed. (Int. 29; patient)

Thus patient views were in contrast to GPs who described concerns that the routine use of the RADTs would encourage increased patient attendance.

\section{Preference for no antibiotics}

Patients reported that they would prefer not to take antibiotics unless needed, and they perceived the RADT as assisting in supporting their no-antibiotic preference, ruling out medication where it is not clinically indicated.

If they need to be prescribed and they can help you, then that's fine but if they're not really going to make any difference then I don't see the point. (Int. 02; patient) 


\section{DISCUSSION}

The study identified three key areas surrounding healthcare practitioners' views on the use of RADTs, these included practicalities relating to the tests; the accuracy of diagnosis provided by the RADT; and patient-related outcomes. Participation in the trial was also identified as strongly influencing attitudes towards the use of RADTs. In addition, patient views on the RADT relating to future GP attendance, antibiotic expectation and reassurance were also identified.

\section{Strengths and limitations}

One of the main strengths of the study is its attempt to include GP, NP and patients to capture a broad view of the RADT in practice. The study was able to directly explore the experience of conducting the RADT as a practitioner and the experience of receiving the test as a patient. This process identified differences in practitioner perceptions of patient attitudes towards the RADT and actual patient views. There was also a relatively large sample size for a qualitative study; however, as the patient sample size was small, further work could usefully focus on this group to confirm and provide additional insight to the present findings. The NP sample was smaller than the GP sample but general consensus in most views was clear within the NP sample and when compared with the GP sample, NPs reported similar views of the RADTs. The only clear difference between the samples was identified in a view which was widely described by GPs but not NPs, that the RADT may be 'unnecessary for experienced practitioners'.

Nesting qualitative interviews in the PRISM trial provided in-depth views, which aided interpretation of the main trial results. In terms of limitations, three researchers conducted the interviews and the level of exploration by each varied. Nonetheless all interviews covered the key questions and this permitted comparative analysis across the data corpus. Transparency of the analytic process and agreement on themes across the team ensured that the final themes were robust.

\section{Main findings}

Overall, interviews suggest that participation in the PRISM trial encouraged a more positive practitioner stance towards the use of RADTs. Positive views towards the RADTs related to patient outcomes such as the test results providing reassurance to the patient that they do not need antibiotics and also providing an educational tool to indicate why antibiotics may not be necessary. Practitioners also described how the test could provide a useful confirmation of diagnosis, which reassured the patient and supported their prescribing decisions. In relation to the practicalities of conducting the RADT, practitioners reported that it was easy to use, possibly a useful tool for less-experienced staff members, and that they felt it had reduced their antibiotics prescriptions for sore throat-hence potentially useful for experienced and less-experienced staff.
There were also some negative views and concerns relating to the RADT. Practitioners' reported concerns regarding the additional costs to the practice and time taken to conduct the RADTs. However, interviews also suggested that concerns relating to time were most likely to be reduced after the practitioner had conducted the tests a few times and had become familiar with the procedure. Concerns were also raised in relation to the accuracy of diagnosis in terms of the possible identification of GABHS carriers who may be unnecessarily prescribed antibiotics, and the possible presence of alternative forms of bacteria which the RADT could not detect. Practitioners reported concerns regarding the difficulty in making a prescribing decision when the RADT result differed from their clinical opinion. However, it was also reported that the RADT could reassure them if they were in doubt over the diagnosis. Finally, there was some concern that the use of RADTS could increase the 'medicalisation' of sore throat and increase patient attendance to the GP for this condition. However, the modest number of patient interviews suggested that tests would be unlikely to increase their GP attendance, and that patients valued the use of the RADT, reporting that they felt reassured by them.

\section{Comparison with existing literature}

It was reported that practitioners viewed the RADTs positively as a tool which could provide a confirmation of their own diagnosis and therefore support their prescribing decision. However, practitioners reported a negative view of the RADT if it presented evidence for a diagnosis that differed from their opinion. Similar findings were reported in a study, which used a computer-based clinical intervention to assist with antibiotic prescribing decisions for respiratory tract infection. ${ }^{12}$ GPs reported acceptance for use of the system if it was perceived as a tool that could support their decisions. However, if the system was perceived as a method of enforcing a prescribing decision, the GPs were more likely to view it negatively and did not wish to use it during their consultations. In similar vein, a study exploring GP views on the use of severity measures for the diagnosis of depression described the measures as beneficial when they confirmed a GP diagnosis and treatment disposal, but were described as of limited value when the score was not congruent with their view founded on clinical experience and intuition. ${ }^{13}$

Practitioners also reported concern that use of the RADT could increase patient pressure for tests and GP consultations for sore throat. However, patients reported that although they felt reassured by the tests it would be unlikely to increase their GP attendance for the condition or their expectation for antibiotics. These findings are analogous to perceptions about prescriptions. GPs often report a perceived pressure from patients to prescribe, investigate and refer despite the fact that patients do not report such expectations. ${ }^{14}$ Findings from a qualitative study of patients attending their general practice 
with suspected urinary tract infection also identified patient reluctance to rely on antibiotics to ameliorate symptoms, while GP interviews highlighted belief in patient pressure or desire for antibiotic prescriptions, ${ }^{15} 16$ suggesting a strong disconnect between patient expectations and practitioner views of patient expectations.

\section{Implications for clinical practice}

Practitioners reported that being in the PRISM trial had resulted in them viewing RADTs more positively than before their participation. It was also reported that GPs who had not taken part in the trial were likely to view RADTs more negatively than their participating counterparts. This suggests that offering GPs the opportunity to pilot or trial the tests before agreeing to implement them in practice could enhance how the RADTs are subsequently embedded into routine primary care. Practitioners who discussed the clinical scoring method widely held the view that it was time-consuming, unnecessary and only beneficial for inexperienced staff. These perceptions may hinder acceptance of the score by experienced clinicians in the future and would need to be addressed in future initiatives aimed at incorporating these tests into routine practice.

Patient views of using the RADTs were generally positive, which suggests that patients would not object to their use in to primary care. A larger sample of patients would be useful to further test out and confirm the acceptability of the RADTs. Practitioners were concerned that the RADT would increase consultations, but patients reported that their GP attendance would remain the same. This finding could be further explored with patients and, if confirmed, could usefully be presented to GPs to reduce their concerns about using RADTs in relation to the effects on patient attendance.

\section{CONCLUSION}

It is unlikely that RADTs will have a place in clinical practice in the near future until healthcare practitioner concerns are addressed, and, importantly, until they have direct experience of using them in real consultations. The routine use of clinical scoring systems for acute upper respiratory illness also faces important barriers related to clinicians' perception of their utility in the face of their clinical experience and intuition.

Contributors PL conceived the idea of the study and was responsible for all aspects of the study including design, interpretation, and commented on drafts and revisions of the manuscript. MM, IW and FH were involved in generation of the study idea, design, and interpretation. LM and GL were responsible for leading the data analysis, interpretation of results and manuscript preparation and revisions. HH, TL, RC were responsible for the acquisition of data, contributed to the interpretation of findings, and approved the manuscript.
Collaborators PRISM Investigators: University of Southampton-Paul Little, Ian Williamson, Mike Moore, Mark Mullee, Man Ying Edith Cheng, James Raftery, David Turner, Jo Kelly, Jane Barnett, Karen Middleton, Lisa McDermott, Geraldine M. Leydon; Tessa Lambton, Rebecca Cooper, Hugo Henderson, James Raftery, David Turner and Rafael Pinedo-Villanueva. University of Oxford—David Mant, Paul Glasziou, Sue Smith, Diane Coulson. University of Birmingham -F.D.Richard Hobbs, Razia Meer-Baloch; Health Protection Agency: Cliodna McNulty, Peter Hawtin.

Funding Funded research only (National Institute for health research (NIHR) Health technology assessment programme). Health technology assessment programme had no role in the conduct or write up of the research.

Competing interests None.

Ethics approval Southampton and South west Hampshire REC (A).

Provenance and peer review Not commissioned; externally peer reviewed.

Data sharing statement No additional data are available.

\section{REFERENCES}

1. Cooper R, Hoffman J, Bartlett J, et al. Principles of appropriate antibiotic use for acute pharyngitis in adults. Ann Intern Med 2001;134:509-17.

2. Snow V, Mottur-Pilson C, Cooper R, et al. Principles of appropriate antibiotic use for acute pharyngitis in adults. Ann Intern Med 2001;134:506-8.

3. Bisno A, Gerber M, Gwaltney J, et al. Practice guidelines for the diagnosis and management of group A Streptococcal pharyngitis. Clin Infect Dis 2002;35:113-25.

4. Sheeler R, Little P. Rapid streptococcal testing for sore throat and antibiotic resistance. Clin Microbiol Infect 2006;12(s9):3-7.

5. Wood F, Brookes-Howell L, Hook K, et al. A multi-country qualitative study of clinicians' and patients' views on point of care tests for lower respiratory tract infection. Fam Pract 2011;28:661-9.

6. NICE guideline development group. Prescribing of antibiotics for self-limiting respiratory tract infections in adults and children in primary care. http://www nice org uk/Guidance/CG69 2008

7. Mclsaac W, Kellner J, Aufricht P, et al. Empirical validation of guidelines for the management of pharyngitis in children and adults JAMA 2004;291:1587-95.

8. Principles of appropriate antibiotic use for acute pharyngitis in adults. http://www acponline org/clinical_information/guidelines 2001

9. Vincent M, Celestin N, Hussain A. Pharyngitis. Am Fam Physician 2004;69:1465-70.

10. Joffe H, Yardley L. Content and thematic analysis. In: Marks D, Yardley L, eds. Research methods for clinical and health psychology. London: Sage, 2004:56-68.

11. Leydon GM, Dowrick CF, McBride A, et al. Questionnaire severity measures for depression: a threat to the doctor-patient relationship? Br J Gen Pract 2011;61:117-23.

12. McDermott L, Yardley L, Little $P$, et al. eCRT Research team: developing a computer delivered, theory based intervention for guideline implementation in general practice. BMC Fam Pract 2010;11:90.

13. Dowrick C, Leydon G, McBride A, et al. Patients' and doctors' views on depression severity questionnaires incentivised in UK quality and outcomes framework: qualitative study. BMJ 2009;338. doi: 10.1136/ bmj.b663.

14. Little P, Dorward M, Warner G, et al. Importance of patient pressure and perceived pressure and perceived medical need for investigations, referral, and prescribing in primary care: nested observational study. BMJ 2004;328:444.

15. Little $\mathrm{P}$, Turner $\mathrm{S}$, Rumsby $\mathrm{K}$, et al. Urinary tract infection: development and validation, randomised trial, economic analysis, observational cohort and qualitative study. Health Technol Assess 2009;13.

16. Leydon $\mathrm{G}$, Turner $\mathrm{S}$, Smith $\mathrm{H}$, et al. The journey from self-care to GP care: a qualitative interview study of women presenting with symptoms of urinary tract infection. Br J Gen Pract 2009; 59:219-25. 